\title{
The Role of Empathy in Making Availability Judgments from Video and Silhouette Awareness Information
}

\author{
Boris de Ruyter ${ }^{*}$, Ehsan Baha ${ }^{2}$, Marten Pij1 ${ }^{1}$ and Panos Markopoulos ${ }^{2}$ \\ ${ }^{1}$ Philips Research Europe, HTC 34, AE 5656 Eindhoven, The Netherlands \\ ${ }^{2}$ Industrial Design, Eindhoven University, Den Dolech 2, 5612 AZ, Eindhoven, The Netherlands
}

\begin{abstract}
This paper reports research concerning video media spaces for the home and specifically the extent to which different approaches for video obfuscation can balance conflicting requirements for awareness between connected individuals and privacy. Different filtering techniques were compared regarding the ability of observers to make inferences regarding the observed, and with regards to the acceptability of being observed through such media. So far, related research has only considered individual differences between observers at a cursory level. We report on two experiments that evaluated whether people ability to empathize with others influences their ability to evaluate the availability of another person based on a video footage. We focused on supporting judgments of availability for communication comparing full video and on silhouette based obfuscation. The first experiment indicated a strong relation between empathy score and availability judgments. This effect was strongest for both males and females in the silhouette visualization condition. To further understand and confirm the effects found in this study, a second experiment involving more test participants and controlling for the correctness of availability judgments was conducted. Our findings suggest that empathetic skills specifically, and social cognition skills more generally, are critical factors for availability judgments.
\end{abstract}

Keywords: Availability management, interruptions, home media spaces, empathy, gender differences, privacy, awareness systems.

\section{INTRODUCTION}

One of the primary benefits for video based media spaces, systems that provide sustained video links between connected individuals, is the resulting awareness of each other's activities and the opportunities they provide for initiating social interactions. Even from the early experimentations with this technology, before it became a viable option for the broader public, the potential tradeoffs related to serendipitous informal social interactions were recognized by researchers [1]. By the same token, awareness of another person may compromise this person's privacy needs. At work, unplanned interruptions may cause undesired interruptions from work; conversely people may be inhibited to initiate contact not knowing when is an appropriate moment to call [2]. In non work settings, mutual awareness can create obligations, expectations or feelings of being monitored, all of which are manifestations of privacy concerns [3].

To address these issues researchers have explored the use of video obfuscation as a means to protect privacy while providing sufficient awareness of each other. There are various algorithms for video obfuscation, e.g., blurring filtration, pixelization. [1, 4-6] aiming initially to guide the application of image processing techniques on 'always on' video, a series of research studies focusing on workplace awareness has examined whether there is a particular level of

*Address correspondence to this author at the Philips Research Europe, HTC 34, AE 5656 Eindhoven, The Netherlands; Tel: +31-40-2747630; Fax: +31-40-2746321; E-mail: boris.de.ruyter@philips.com filtration in which either pixelization or blurring would allow a sufficient level of awareness cues to be presented that will support inferences relating to the observed person's availability. For example, [1] examined the use of these techniques for connecting office workers, showing that some promise as to the use of video distortion techniques: at some medium level of filtering, it appears to possible to provide sufficient awareness while people expect that their privacy will be protected.

Similar issues arise when considering the application of media spaces in domestic environments. [7] found that evaluating each other's availability for communication is one of the most pronounced awareness needs for young working parents. Awareness can provide social connectedness and can prompt direct communication [8]. However also in the domestic domain, awareness can give rise to privacy concerns. [4] compared pixelization and filtering as ways to obfuscate video. Contrary to the workplace media spaces they found that at the levels of filtering where sufficient information would be presented to allow availability judgments to be made, participants reported feeling uncomfortable regarding their privacy. Their conclusion was that video obfuscation is not a sufficient means to protect privacy at home environments. These findings are placed within the context of a teleworking scenario, where the home occupant has a permanent video connection with their work. The availability of a permanent connection means that quite personal moments can be captured potentially on video; indeed [4] focused on privacy concerns regarding 'risky' moments, e.g., when the home occupant would appear naked on video. As one might expect, their investigation showed 
that attitudes regarding privacy risk differ very much depending on the situation captured on video.

In this research we consider the use of video to capture less threatening everyday situations and especially with the aim to provide awareness and connectedness between family members [9]. We are interested in allowing friends and family to obtain awareness of each other, and especially to make availability judgments regarding the initiation of direct communication. In an experimental study that considered the potential benefits of domestic media spaces to connect friends and family, [10] evaluated a specific form of obfuscated video; silhouette images were used to portray movements of people in front of the camera. When connecting related people engaged in similar social activities (without any of them being perceived as personal or secret in any way), participants felt high social presence of each other and had increased group attraction. In this research we evaluate the use of silhouettes as a video obfuscation technique by comparing it to providing full video imagery.

The aims of this study are rather different than the earlier studies of video filtration discussed above. Rather than seeking the sweet spot regarding the level of video filtration where privacy and awareness needs are both met, (as was the intention for [4]), we are more concerned with individual differences regarding the ability of people to draw inferences from such video images.

This issue has been largely untouched by the earlier studies reported above; [4], report some differences regarding the attitudes of men and women regarding the privacy threat that a filtrated video represents and speculated that different attitudes might relate to personality differences. However, no systematic investigation of this issue has been done to date, neither has there been any attempt to evaluate individual differences regarding the inferences made from video as such, nor has there been an attempt to relate these to specific skills of individuals in assessing social situations.

The remainder of this paper presents an experiment that evaluated how gender and empathy influence the interpretation of video images shown, comparing full video and silhouette-based obfuscation. The role of empathy in evaluating social situations is discussed, the set up of the experiment and its results are described, concluding with a discussion that examines implications for future work.

\section{EMPATHY}

By nature humans develop from isolated and egocentric newborns into socially engaging human beings. The process of social development involves a separation from the self and the environment which is instrumental in the development of personality and autonomy. This development is driven by social interest: a need to position ourselves in, and differentiate ourselves from, the social environment. In his "Individual Psychology", Alfred Adler [1] discusses how social interest drives people to connect socially to society as a way of dealing with an inferiority complex that characterizes the social development of human beings. Obtaining a position in society will deliver us status and recognition and thus help us in coping with this inferiority complex. Or, as Schutz [11] argues in his "Interpersonal Needs Theory": the drive to develop interpersonal relationships and communicate with others is based on the need to feel accepted, understood, and valued.

During our social development it is essential that we develop empathic skills to exploit social relations in a manner that is acceptable for our social environment. Empathy is described as the intellectual or imaginative apprehension of another's condition or state of mind without actually experiencing that person's feelings [12]. However, empathy is also a mechanism that can be exploited in technology-supported social interactions. Information or footage on others, their context, and activities need to be interpreted and understood and placed within the context of the social interaction between individuals. Earlier attempts to operationalize affective benefits and costs of communication [3] through systems like media spaces or awareness system, have illustrated the relevance of the apprehension of the situation of the communicating party: appreciating expectations raised, whether the other feels obliged to call, whether the other feels involved in one's life, etc. This perspective taking clearly is founded upon an individual's interpersonal skills. Specifically we expect that individuals will use their empathic skills to interpret video footage representing situations in which a remote person is engaged.

Below, we report on two experiments into the use of silhouette visualizations of awareness information for judging availability. While the first experiment seeks to discover potential interaction effects between empathy and availability judgments, the second experiments is aimed at further exploring these effects with a larger sample size.

\section{EXPERIMENT 1}

In this experiment the relationship between empathic skills and availability judgments of actors in video clips of typical home situations is studied; N.B we are not per se interested in the accuracy of the availability judgments but on the impact empathy could have upon them.

\section{Method}

\section{Participants}

The experiment involved 44 voluntary and unpaid participants (with an average age of 35 years) who were recruited in both an academic and an industrial research organization. The experiment was conducted using an online system that participants recruited by email postings, accessed through a dedicated website.

\section{Materials}

The Empathy Scale [13] was used to obtain a general empathy score from the participants. This 30 item questionnaire provides empathy scores that load on six subfactors and a general empathy factor. While this instrument has been validated, it does not provide normative data. However, for the present experiment this is not a problem since we want to compare empathy scores between experimental conditions (rather than making normative interpretations about the empathic scores).

The contents of the video clips represent the following typical home situations:

1. Empty room 
2. Leaving the room

3. Entering the room

4. Having dinner

5. Resting on the coach

6. Reading a magazine

7. Watching television

8. Making a phone call

9. Having a person to person conversation

While the "empty room" and "leaving the room" situations were chosen as a baseline for non-availability of the remote person to engage in a phone conversation with the participants, it is known that transition situations (e.g. entering or leaving the room) elicit clear availability judgments [14]. The other five situations are representative for a typical home context and, as mentioned in our introduction, avoiding personal/private activities such as undressing or taking a shower.

The situations were presented as 20 -second video clips with a 200x200 pixel resolution. Research has indicated that the minimum resolution for availability judgments based on video material should have a resolution of at least $128 \times 128$ pixels [14]. The video clips could be either a full video visualization or silhouette type visualization. The silhouette visualization is realized by representing the activity captured on the video by the contours of moving objects only. The actors in the video clips are not related to any of the participants and there are no additional contextual cues such as time of the day available in the video clips themselves.

A limitation of conducting an experiment where prerecorded video clips are viewed compared to the actual situation we wish to project our results upon, is that in the latter, the time of the day is known to the viewer. E.g., if someone is eating and it is early in the morning, they can be assumed to have breakfast without actually being able to see what this person is eating. This issue has not been addressed in the experimental set ups of earlier studies discussed above; to evaluate the impact of such salient contextual information upon our dependent measures, we also provided to some participants this information as a textual cue under the video image

\section{Experimental Design}

A $2 \times 2$ between-subjects experimental design was adopted. Participants were assigned to one of four conditions (11 participants per condition):

\begin{tabular}{|l|l|l|}
\hline & Full Video & Silhouette Video \\
\hline No Contextual Cues & Video & Silhouette \\
\hline Additional Contextual Cues & $\begin{array}{l}\text { Video }+ \text { time of } \\
\text { the day notice }\end{array}$ & $\begin{array}{l}\text { Silhouette }+ \text { time } \\
\text { of the day notice }\end{array}$ \\
\hline
\end{tabular}

In the first two conditions participants were asked to base their availability judgment either on full video visualizations or on silhouette visualizations of the same situations. In the second two conditions, the participants would also get an indication of the time of the day in which this situation is taking place.

\section{Procedure}

After acknowledging the test instructions and signing the informed consent form, participants were asked to complete the Empathy Scale. Depending on the experimental condition, participants were asked to watch and rate on a five point scale (in terms of availability for engaging in a phone conversation with the persons in the video) a set of nine fullvideo or silhouette visualizations with or without additional contextual cues (e.g. the time of the day). The order of the presented clips was randomized across participants.

The clips would start playing automatically and participants could not proceed to view the next clip without providing a rating regarding availability for the current clip. Participants could choose to replay a video as many times as they wished to (as indeed was the case in the earlier studies reviewed above). The availability question is phrased as: "After having seen the video, how would you judge the availability of the person(s) (based on their activity) to engage in a phone conversation with you?"

\section{Results}

In total 26 males and 18 females completed the experiment. Statistical data analysis was performed on the Empathy and Availability scores. When asked to describe the situation represented in the full video or silhouette video, all participants (albeit in different wordings) recognized the nine situations. Some participants also provided potential reasons for the context (e.g. "the person has gone to sleep" for the empty room situation).

\section{Empathy Scores}

Using an unpaired t-Test we found a significant $(\mathrm{p}<$ 0.01 ) difference in Empathy scores between male and female participants: female participants in this study demonstrated a significant higher empathy score than males.

\section{Availability Scores}

A two-tailed Man-Whitney test (signed ranked sums) was used to test differences in availability judgments scores between the full video and the silhouette video visualizations shown to study participants.

Visualization type. Other than for the Eating $(\mathrm{p}<0.04)$ and Leaving $(\mathrm{p}<0.01)$ situations, there are no statistical significant differences in availability scores between the full video and silhouette video visualizations.

Contextual cues. When the additional context cue of time is given, there is a significant difference for the Away $(\mathrm{p}<0.01)$ and Arriving $(\mathrm{p}<0.02)$ situations. For all other situations there is no statistical significant difference in availability scores.

These results mean that the use of filtration does influence an individual's ability to make availability judgments based on the video clips shown but only for the Eating and Leaving situations; this seems sensible as the nature of these activities becomes clearer in full video than in silhouettes. The Away scene where the silhouettes filtration would show no activity at all (since nobody is in 
the room) requires a contextual cue to be meaningful as a full video and one can expect that it provide significantly less cues regarding availability in the silhouette condition.

\section{Availability by Empathy Scores}

Using the availability scores as predictors for the empathy score, we can perform a regression analysis on the obtained scores ${ }^{1}$. Since the experiment involved a small sample $(n=44)$ we used the adjusted R-square ${ }^{2}$ for quantifying the amount of variance explained. Given the significant difference in empathy scores between male and female participants, we will conduct regression analysis for both groups separately.

Male participants. Considering the availability judgments for both the full video and silhouette video, we can explain $42 \%$ of the variance in the availability judgments from the empathy scores. When considering only the silhouette video a total of $82 \%$ of the variance in the empathy scores can be explained on the basis of the availability scores.

Female participants. Using availability scores for both visualization types together in the regression analysis, 55\% of the availability's score variance is explained from the empathy scores. When using the silhouette video data for females in this study in a regression analysis $98 \%$ of the availability's variance is explained.

Though these results do not say anything regarding the veridicality of availability judgments, they show clearly that an individual's empathic ability has a higher impact on their ability to make availability judgments when filtration is applied and has a more pronounced influence on the availability judgment for women than for men.

\section{Discussion}

It is interesting to note how accurately participants were able to identify the activity shown on both video and silhouette conditions. Contrary to the studies discussed of pixelization and blurring by [4], there were not different levels of obfuscation evaluated: the silhouette visualization was either used or not. Still, given that the imagery shown does by its nature conceal images that one might not want to be shown on video, this result indicates that this technique can reach the compromise between the needs for awareness and privacy in video media spaces that related research studies have been looking for.

The finding that contextual cues (time in this case) are important for the Away and Leaving situations seems logical since contextual cues such as time of the day give a completely different interpretation to the situation. Indeed, as some participants indicated: a person leaving the room late in the evening might suggest that this person is going to bed and meaning it is not such a good moment to give the person a phone call.

Although there is no normative data available to interpret the Empathy scores, it is noted that female participants in this study demonstrated a significantly higher level of

\footnotetext{
${ }^{1}$ The use of regression analysis with ordinal level data such as obtained with Likert scales does not significantly affect Type I and Type II errors.

${ }^{2}$ The adjusted R-square value is calculated as $1-(1-\mathrm{R}$-square $) *(n-1) /$ $(n-p-1)$.
}

empathy than males. Also, for females there was found a stronger relation between empathy score and availability judgments in the silhouette visualization condition. This effect was strongest for both males and females in the silhouette visualization condition. This finding confirms our expectations that individual differences and especially those relating to personal skills play an important role in how effectively video and filtered video can be used for making availability judgments. This issue, previously ignored by related studies, merits further investigation. In particular, we sought out to examine its impact upon the accuracy of the availability judgments made on the basis of the video.

\section{EXPERIMENT 2}

While the previous experiment provided some interesting insights into the relationship between empathy and availability judgments, it does not control for the correctness of the availability judgments. Given that the video clips in the previous experiment were acted by an actor, there was no criterion to judge the validity of the participant's availability judgment.

Moreover, experiment 1 as well as the similar studies for work and domestic contexts discussed in the introduction, can all be criticized regarding the artificiality of the situation presented. One could argue regarding the representativeness of the videos shown and especially regarding the extent to which people will be called to make availability judgments based on such video imagery. In this follow-up experiment we will focus on the relationship between empathy scores and availability judgments that are controlled for their accuracy and seek confirmation for the potential effects found in the previous experiment.

In this experiment we are thus using Empathic Accuracy as independent variable. The term Empathic Accuracy [15] was introduced to describe a person's ability to accurately infer what another person is thinking or feeling.

In order to conduct a more reliable statistical analysis of the findings, this experiment will focus on the silhouette visualization condition only and involve significantly more test participants than in the previous experiment.

\section{Method \\ Participants}

For this experiment we recruited 46 voluntary and unpaid participants (with an average age of 26 years) in both an academic and industrial research organization. These participants did not participate in the previous experiment. Since the experiment was conducted using the same online system as in the previous experiment, participants were also invited via email and provided with an URL of the experimental setup.

\section{Materials}

While the same scenarios as used in the previous experiment are reused in this experiment, the videos are now recorded in real life settings. Video records were made at different times of the day and week at the homes of 20 individuals as they go about their daily activities. From each individual a subset of clips were selected from the footage for which we obtained permission to use as stimuli in our 
experiment and for which we asked the person to rate their own availability for a phone communication at that moment when the video was recorded. For this, we used the same availability scale as in the previous experiment (this rating was done immediately after recording the video clip). As in the previous experiment these videos were transformed into silhouette representations to be used in the actual experiment. We selected video clips to represent the same set of situations as in the previous experiment, only in this case they showed actual situations for which we could have ground truth regarding the availability of the person shown.

\section{Experimental Design}

Since our main interest in this experiment is to assess the relationship between empathy and availability judgments in silhouette visualizations (controlled for the correctness of the judgment), the experimental design consists of only one condition. In this condition test participants will watch each silhouette video clip and make a judgment of the availability (to engage in a phone conversation) of the person in the clip. In contrast to the previous study, the time of the day was not given in this experiment.

\section{Procedure}

The methodology adopted in this experiment follows the research methodology for measuring empathic accuracy as introduced by [16]. In this methodology a target person is being video recorded. At a specific point the recording is stopped and the target person is asked to provide what this person is feeling or thinking. In our study the question was obviously if the person would be available for engaging in a conversation with a remote person. During the study, the test participants will watch this video and are required to indicate if the target person would be available to engage in a conversation at the point where the video is stopped. The experimental procedure for data collection was similar to the procedure followed in the previous experiment and consisted of test participants going through the procedure for watching and evaluating video clips.

\section{Results}

The raw availability scores obtained from the experiment were corrected using the 'ground truth' collected during the recording of the video clips. Although the availability scores are collected on a 5 point scale, we recoded the collected data to a correct or incorrect judgment by ignoring the spread over the extremes of the scale. In other words, we did not differentiate between a score "completely available" and "available": both would be interpreted as "available" (both for the actors in the videos and the test participants) and similar for the "unavailable" scoring. So the data analysis was conducted on "correct/incorrect" scores based on the judgments "available/don't know/unavailable". Further data analysis will be performed on the scores indicating the correctness of the availability judgment.

Before conducting the statistical data analysis we verified the distribution of the Empathy scores in the data set using the D'Agostino [17] test for normality. This normality test is based on the skewness coefficient and indicates that our Empathy score distribution does not significantly deviate from a normal distribution $(\mathrm{p}=0.45)$.
Given the unbalanced proportion of males and females in this sample (10 females) we cannot test for gender effects as was done in the previous experiment.

Similar to the data analysis in the previous experiment, we conducted a regression analysis. The regression analysis (based on $\mathrm{n}=46$ ) provided a solutions that explains almost $88 \%$ of the variance in the data set (adjusted R-square $=$ 0.878 ).

Furthermore, this regression solution is significant $(\mathrm{F}=$ $37.91, \mathrm{p}<0.001)$. The scenarios "Watching television" and "Having a person to person conversation" provided most correct availability judgments while "Having dinner" provided the least correct judgments.

\section{Discussion}

The results of experiment 2 confirm the findings of the first experiment: empathy plays an important role in making availability judgments from silhouette representations. Additionally, this experiment takes the correctness of the availability judgments into account: empathy scores are a good predictor of the correctness of the availability judgment made. This result is as we expected; observers with higher empathy are more capable of taking the perspective of the observed, and to evaluate whether this person is available for communication or social interruption or not. While expected, this result is quite novel in that previous research has not attempted to examine what individual characteristics influence availability judgment and influence the effectiveness of video obfuscation mechanisms. Further it points to the need for considering social skills of individuals and how to support them when designing applications for mediated social interaction.

\section{CONCLUSIONS}

The current study has found evidence for an interesting trend in the relationship between empathic skills and availability judgments from awareness information.

Our analysis of the empathy scores of our participants is in line with the earlier findings by [18] that women exhibit more empathy while interpreting awareness and presence information. Differences in empathic accuracy between women and men have been explained by various factors including awareness of one's own empathic ability [19, 20], the gender of the target person [21], the relationship with the target person [22], or the physical attractiveness of the target [16].

In a more recent study, [21] have indicated that there is no difference in empathic ability between men and women but rather a motivational difference that accounts for different empathic ability exhibited. Once scores are corrected for this motivational aspect, men were found to exhibit as much empathic abilities as women. However, since our study did not introduce any mechanisms to influence the participant's motivation, we suspect that yet other mechanisms influenced the gender difference established in our study.

That empathic skills play an important role in making availability judgments from awareness information such as silhouette visualizations is intriguing. At the very least it illustrates that individual differences should be considered 
by this area of research that examines availability management (and video media spaces more generally) as they can represent significant confounds for empirical findings. Looking more broadly than empathy as such, research needs to consider people's ability, to judge others' feelings, thoughts, attitudes, intentions in a particular social context; such capacities of individuals pertain to their social intelligence [23]. While it seems quite logical that this broader perspective on human capabilities should be brought to bear upon the investigation of media spaces, they have been largely ignored by research so far.

From a design perspective the results presented seem indicate that higher priority should be placed on supporting through communication media people's empathic skills and, more generally, their social intelligence.

A related design approach by [24] focuses on allowing people to carry over social skills from their daily life to their mediated social interactions, a point also argued by [25] in relation to groupware systems in general. However, such research has not gone as far as to classify and describe concretely the social skills that one would expect to support in a particular situation. The current study would suggest the use of contextual cues in awareness and presence applications that elicit strong empathic feelings with endusers.

Considering the design space of awareness systems [26] this would favor using video imagery rather than abstracted information regarding individuals, it would favor expressiveness rather than just conveying factual information efficiently. Future investigations are aimed at evaluating the relevant value of abstract information assembled through context sensing technologies versus the use of video media spaces in the home environment. We expect that they represent a different trade-off regarding privacy and awareness as discussed above, but on the grounds of the present study, that media spaces are a more appropriate medium for people to deal with socially challenging interactions and make appropriate judgments.

\section{REFERENCES}

[1] Boyle M, Edwards C, Greenberg S. The Effects of Filtered Video on Awareness and Privacy. In Proceedings of the ACM Conference on Computer Supported Cooperative Work - ACM CSCW'00. ACM Press 2000; pages 1-10.

[2] Cockburn A, Greenberg S. Making contact: getting the group communicating with groupware, Proceedings of the conference on Organizational computing systems 1993; p.31-41. Crowley JL, Coutaz J, Bérard F. Perceptual user interfaces: things that see. Commun ACM 43(3): 2000; p.54.

[3] IJsselsteijn W, Baren J, Markopoulos P, Romero N, de Ruyter B. Measuring Affective benefits and costs of mediated awareness: development and validation of the abc-questionnaire. In: Markopoulos P, De Ruyter B, Mackay W, Eds. Awareness systems. Springer London 2009; pp. 473-88.

[4] Neustaedter C, Greenberg S, Boyle M. Blur filtration fails to preserve privacy for home-based video conferencing. ACM TOCHI 2006; 13(1): 1-36
[5] Zhao QA, Stasko JT. Evaluating image filtering based techniques in media space applications Proceedings of the 1998 ACM conference on computer supported cooperative work 1998; pp.118.

[6] Crowley JL, Coutaz J, Bérard F. Perceptual user interfaces: things that see. Commun ACM: 2000; 43: p. 54.

[7] Khan V, Markopoulos P. Busy families' awareness needs. Int J Hum Comput Stud 2009; 67(2): 139-53.

[8] Romero NA, Van Baren J, Markopoulos P, De Ruyter B, Ijsselsteijn WA. Addressing interpersonal communication needs through ubiquitous connectivity: home and away. Proceedings of the first european symposium on ambient intelligence. Springer 2003; pp. 419-29

[9] Markopoulos P, Romero N, van Baren J, IJsselsteijn W, de Ruyter B, Farshchian: B. Keeping in touch with the family: home and away with the ASTRA awareness system. CHI Extend Abst 2004: 1351-4.

[10] De Ruyter B, Huijnen C, Markopoulos P, Ijsselsteijn WA. Creating social presence through peripheral awareness. Proceedings of Human Computer Interaction International; Crete, Greece. June 22$27,2003$.

[11] Schutz WC. FIRO: A three dimensional theory of interpersonal behavior, New York: Holt, Rinehart \& Winston 1958.

[12] Hogan R. Development of an empathy scale, J Consult Clin Psychol 1969; 33: 307-16.

[13] Mayer JD, Caruso DR, Salovey P. Emotional intelligence meets traditional standards for an intelligence. Intelligence 2000; 24 (4): 267-98.

[14] Johnson B, Greenberg S. Judging people's availability for interaction from video snapshots. Proceedings of the Hawaii International Conference on System Sciences January 1999; 5-8.

[15] Ickes W. Empathic accuracy. J Pers 1993; 61: 587-610.

[16] Ickes W, Stinson L, Bissonnette V, Garcia S. Naturalistic social cognition: Empathic accuracy in mixed-sex dyads. JPSP 1990; 59: 730-42.

[17] D'Agostino RB, Belanger A, D'Agostino RB, Jr. A suggestion for using powerful and informative tests of normality. TAS 1990; 44(4): 316-21.

[18] Nicovich SG, Boller GW, Cornwell TB. Experienced presence within computer-mediated communications: Initial explorations on the effects of gender with respect to empathy and immersion. ACM Trans Comput Hum Interact 2005; 10(2): article 6.

[19] Eisenberg N, Lennon R. Sex differences in empathy and related capacities. Psychol Bull 1983; 94: 100-31.

[20] Graham T, Ickes W. When women's intuition isn't greater than men's, In: Ickes W, Ed. Empathic accuracy. New York: Guilford 1997; pp. 117-43.

[21] Klein KJK, Hodges SD. Gender differences, motivation and empathic accuracy: When it pays to understand. Pers Social Psychol Bull 2001; 27: 720-30.

[22] Simpson JA, Ickes W, Blackstone T. When the head protects the heart: Empathic accuracy in dating relationships. J Pers Social Psychol 1995; 69: 629-41.

[23] Vernon PE. Some characteristics of the good judge of personality. J Social Psychol 1933; 4: 42-57.

[24] Erickson T, Kellogg WA. Social translucence: an approach to designing systems that support social processes. ACM Trans Comput Hum Interact 2000; 7(1): 59-83.

[25] Grudin J. Groupware and social dynamics: eight challenges for developers. Commun ACM 1994; 37(1): 92-105.

[26] Markopoulos P. A Design Framework for Awareness Systems. In: Markopoulos P, de Ruyter B, Mackay W, Eds. Awareness systems: advances in theory, methodology and design. Hum Comput Interact 2009; Part I: 49-72. 